\title{
Cell cycles explained
}

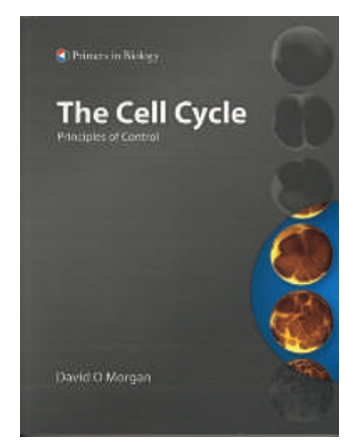

\section{The Cell Cycle: Principles of Control}

\section{Edited by David Morgan}

Oxford University Press $\bullet 2006$

$£ 27.99 / \$ 49.95$

\section{Reviewed by}

Christina Karlsson Rosenthal

The fundamental problem of how cells reproduce has been studied intensely ever since dividing cells were first observed by microscopy. Since, the discovery in the seventies and eighties of the core machinery regulating the eukaroytic cell cycle, the cyclin-dependent kinases (cdks) and cyclins, the cell-cycle field has grown enormously. To identify and organize the major findings in the field is a challenge that David Morgan has taken on in his new textbook. As stated by the author in the foreword, the goal of the book is to provide a coherent framework that covers the key problems in cell division. Thus, it centres on how external and internal signals control the activity of the cyclin-cdk machinery, and how this machinery in turn governs DNA replication and chromosome segregation. Given the vast body of information in the field, it's not surprising that the book took six years to complete. Summarizing and presenting the current knowledge on the cell cycle in a clear and comprehensive way is a formidable achievement.

This well-structured and accessible textbook begins with an introduction to the general concept of discrete cell-cycle phases and basic mechanisms of control in the first chapter. Work in yeast and in Xenopus embryos was key to identifying the basic cell-cycle components and their regulators. These, and newer model systems (newer that is, for cell cycle researchers) such as C. elegans and Drosophila, are briefly introduced in chapter 2 before the multiple regulators of cdk activity and their role in different cell-cycle phases are extensively discussed in chapter 3 . Although the pedagogical need to present simplified models does not always allow discussion of differences between organisms, these are sometimes mentioned - such as the speciesspecific requirement for different cyclins in replication initiation. Chapter 4, named 'chromosome duplication', also covers relatively new areas of investigation, such as how chromatin structure is preserved during cell division.

A quarter of Morgan's book, from chapters 5-8, describes the control of mitosis and cytokinesis, and is devoted to discussing how replicated chromosomes condense and how the mitotic spindle forms, how

Christina Karlsson Rosenthal is Associate Editor at Nature Cell Biology, 4 Crinan Street, London N1 9XW, UK.

e-mail: c.karlsson@nature.com aligned sister chromatids are pulled to opposite poles of the cell and how nuclei are physically separated by the contractile ring to form the two daughter cells. Here, different cyclin-cdk complexes cooperate with other mitotic regulators (such as Polo- and Aurora kinases) to coordinate the architectural changes in the cell. A focused presentation and many schematic drawings (for example, of microtubule behaviour at various steps of spindle function) help the reader understand these complex and dynamic processes. Aspects of meiosis, such as the important mechanism of homologous combination to create genetic diversity, are discussed in chapter 9.

The in-depth description of all the essential aspects of the cell cycle makes this book a unique resource.

The irreversible commitment made in G1 phase to enter a new cell cycle is described in chapter 10, which also covers what is known about the coordination between cell growth and cell division. The faithful replication and segregation of the DNA is dependent on the existence of checkpoints that monitor the accuracy of these events and prevent cell-cycle progression when problems arise. Accordingly, the DNA-damage response is extensively reviewed in chapter 11 . Finally, the clinical relevance of studying the cell cycle is evident from the last chapter, which deals with how accumulated mutations lead to the unrestricted growth of cancer cells.

The in-depth description of all the essential aspects of the cell cycle makes this book a unique resource. Each of the 12 chapters begins with an overview before going into details, which is useful for the uninitiated reader. The book belongs to the "Primers in biology" series, where modular organization of the content allocates two pages to each topic. This organization provides quick access to a specfic topic of interest (for example, 'protein degradation in cell-cycle control' or 'the kinetochore') and despite the two-page limit, a surprising level of detail is provided. Having a single author has kept redundancy to a minimum and has ensured coherence and extensive cross-referencing between chapters. References are provided both to review articles and primary research papers. Key words are defined in each section, but also are collected in a glossary at the end. Illustrations are plentiful and of high quality, and the chapters on mitosis and cytokinesis, in particular, benefit from beautiful immunofluorescence microscopy images.

This is an extremely useful book, which can be used in undergraduate (for an advanced course) and graduate teaching, and as a reference book for researchers in the field. The online resource provides access to all colour illustrations in the book. In addition, anyone teaching a course can also get free online access to the text and to updates. Such updates should be useful, as the only obvious drawback with the book is that some sections will inevitably become outdated quickly because of the energetic pace of research. Regardless, the book will remain an essential source of information for curious students and scientists for some time.

COMPETING FINANCIAL INTERESTS

The author declares that she has no competing financial interests. 